\title{
Social Appearance Anxiety in Patients with Contact
}

\section{Dermatitis}

\author{
Faruk Kurhan \\ Department of Psychiatry, Faculty of Medicine, Van Yuzuncu Yil University, Turkey, Van
}

\begin{abstract}
Contact dermatitis is a dermatological disease that can cause skin lesions on visible and invisible parts of the body. Lesions in the visible parts of the body can negatively affect the body perception of people and cause them to worry about their appearance. The aim of this study was to evaluate the social appearance anxiety of individuals with contact dermatitis lesions on the visible parts of their bodies using the Social Appearance Anxiety Scale (SAAS) and to compare them with healthy controls.

A total of 129 patients aged between 18-65 years with a diagnosis of contact dermatitis and 89 healthy controls were included in the study. Patients and healthy controls were asked to fill in Social Appearance Anxiety Scale (SAAS), Hospital Anxiety Depression (HAD) scales, and dermatological quality of life and visual analog scales were applied to the patient group.

In terms of the mean Social Appearance Anxiety Scale (SAAS) scores, the mean SSI score in the patient group was 57.11, while the mean SSI score in the control group was 19.27. Thus, the mean SDSS score of the patient group was found to be considerably higher than the control group $(\mathrm{p}<0.01)$. A significant positive correlation was found between SSQ score and VAS score $(r=0.282, \mathrm{p}<0.05)$. Lesions appearing in visible places in contact dermatitis patients cause anxiety in individuals due to their appearance. This situation revealed that those with contact dermatitis lesions should receive psychiatric treatment in addition to dermatological treatments.
\end{abstract}

Keywords: Contact dermatitis, Social appearance anxiety, Anxiety

\section{Introduction}

Contact dermatitis is a localized inflammatory skin reaction to various chemical or physical agents (1). The clinical presentation varies depending on various factors such as the characteristics of the irritant, strength, dose, exposure time, frequency, environmental factors and skin sensitivity(1). The most common type is allergic contact dermatitis. It accounts for $80 \%$ of occupational contact dermatitis, is considered the most common cause of hand eczema, and is more common in women than men (2).

Dermatoses such as contact dermatitis, eczema, and psoriasis are classified in the group of psychosomatic diseases that can be exacerbated and worsened by emotional stress (3). When the literature is reviewed, factors such as anxiety, depression and stress affect the severity of dermatological diseases (4). On the other hand, these factors can also cause some skin diseases (5). The role of psychological factors is pointed out at the beginning of the most important factors in the recurrence of resistant dermatoses and the persistence of the symptoms (6).

Therefore, psychodermatology is divided into 3 categories according to the relationship between skin lesions and mental disorders (5).

i) Psychophysiological disorders not associated with mental disorders (Psoriasis, eczema)

ii) Psychiatric disorders causing primary skin diseases (trichotillomania) iii) Skin diseases (ichthyosis, vitiligo) that can cause bodily perception disorders, secondary psychiatric disorders that cause anxiety, depression, fear and suicidal thoughts

In this classification, behavioral or mental disorders related to deterioration in quality of life can be added in patients with chronic dermatosis. As a result, the affected patients may develop avoidance behavior and may have problems in social activities such as shaking hands with someone they meet in social situations (7) .

Undoubtedly, the most important tissue in the formation of the body image perception of individuals is the skin. The physical appearance of the skin significantly affects individuals. A healthy skin plays an important role in protecting the physical, mental, social and occupational health of individuals. It is important for the attractiveness of the visible parts of the bodies of individuals to the opposite sex (8). Ultimately, dermatoses, especially on the face, hands and other visible places, can lead to deterioration of physical and mental health, decrease in self-esteem, difficulties and deterioration in social and professional interaction (9).

Social appearance anxiety disorder is a condition characterized by intense, irrational, and persistent fear and anxiety from situations that require one or more social performances, such as meeting someone new in social situations, speaking in public or in a group, or eating or drinking in crowded environments where one thinks they are observed. Affected individuals are extremely afraid of being negatively evaluated or humiliated or humiliated by 
others and exhibit irrational avoidance behavior in order to avoid these situations, which brings about isolation from society (10).

Lesions that exist in the visible parts of the body in contact dermatitis patients may cause difficulties in their lives, loss of workforce, difficulties in social relations, and damage in family relations, since they may cause social appearance anxiety. When we look at the literature data, we could not find any publication that investigated anxiety disorder due to social appearance in contact dermatitis patients. It was aimed to measure social appearance anxiety in patients diagnosed with contact dermatitis in patients with visible lesions, to compare with the control group, and to evaluate the relationship of social appearance anxiety with anxiety symptoms, depression symptoms, disease severity and dermatological quality of life.

\section{Materials and Methods}

This study is a descriptive cross-sectional study. Volunteer patients with contact dermatitis, especially in the visible areas of their bodies, were included in the study in the Medical Faculty at Van Yuzuncu Yil University Hospital Medical Faculty Dermatology outpatient clinic. Individuals over the age of 18 and under the age of 65 were included in the study. The scales were applied to literate individuals who were at least primary school graduates. Individuals who do not currently have any other psychiatric or skin disease were included in the sample.

The Social Appearance Anxiety Scale (SAAS) was created to measure anxiety about being negatively evaluated by others because of one's overall appearance, including body shape. The scale, developed by Hart et al., (2008) and adapted to Turkish by Doğan (2011) (11), consists of 16 items and one dimension. The SAAS Cronbach's Alpha internal consistency coefficient was .91 and the testretest reliability coefficient was .80 .

The aim of this study was to evaluate the validity and reliability of hospital Anxiety and Depression (HAD) Scale in medically ill patients. This scale was developed by Zigmond and Snaith. There are 14 questions in the scale filled by the patients themselves. While seven of the 14 questions measure the level of depression, the other seven questions measure the level of anxiety. The questions measure four-point Likert-type. The Turkish validity and reliability study of the scale was carried out by Aydemir et al (12).

Sociodemographic Data Form, Social Appearance Anxiety Scale (SAAS) (11), Hospital Anxiety Scale (HAD) (12), Visual Analogue Scale (VAS) and Dermatological Quality of Life Index (DLQI) (13) were applied to the patient group. Sociodemographic Data Form, Social Appearance Anxiety Scale (SAAS)
(11), Hospital Anxiety Inventory (HAD) (12) scales were applied to the control group.

The necessary written permissions for the study were obtained from the Medical Faculty at Van Yuzuncu Yil University Hospital non-interventional ethics committee with Decision number 2020/02-7 and dated 21/02/2020. Consent was obtained from the participants in terms of "informed consent principle" and "volunteering principle".

Statistical Analysis: Descriptive statistics for the continuous variables were presented as Mean, Standard deviation, minimum and maximum values while count and percentages for categorical variables. Normality assumption of the continuous variables was tested with Kolmogrov-Simirnov test. After normality test, Independent $\mathrm{t}$ test was used to compare group means. For determination linear relationships among the variables, Pearson correlation analysis was carried out. Statistical significance level was considered as 5\% and SPSS (ver: 21 ) statistical program was used for all statistical computations.

\section{Results}

A total of 129 contact dermatitis with a mean age of 26.10 (birim) and 89 healthy control individuals with a mean age of 25.22 (birim) were included in the study. Gender, marital status, educational status, occupation and other sociodemographic data of the groups are given in Table1.

SAAS values were found to be significantly higher than the control group. $(\mathrm{p}<0.05)$. On the other hand, HAD anxiety and depression subscales were higher in the control group.

The difference between the patient and control groups in terms of mean SAAS scores was found to be statistically significant. The mean SAAS score in the patient group was 57.11 while the mean SAAS score in the control group was 19.27. Thus, the mean SAAS score of the patient group was found to be quite high compared to the control group $(\mathrm{p}<0.01$.

There was no significant difference between the patient and control groups in terms of HAD anxiety subscale scores. Average in the patient group HAD anxiety subscale scores of 6.96 , while the mean in the control group HAD anxiety subscale scores were found to be 7.10 (Table 2).

Average HAD depression subscale The difference between the patient and control groups in terms of scores was found to be statistically significant. Average in the patient group HAD depression subscale scores were 6.31, while the mean in the control group HAD depression subscale scores were found to be 7.2921. Thus, the average of the patient 
Table 1. Sociodemographic Clinical Characteristics of the Participants

\begin{tabular}{lccc}
\hline & & Number & percentage \\
\hline \multirow{2}{*}{ Group } & Patient & 129 & 59.17 \\
Gender & Control & 89 & 40.83 \\
& Male & 89 & 40.83 \\
Marital status & Female & 129 & 59.17 \\
& Single & 116 & 53.21 \\
Educational Status & Married & 102 & 46.79 \\
& Primary School & 65 & 29.82 \\
& High School & 16 & 7.34 \\
Occupation & University & 137 & 62.84 \\
& Housewife & 48 & $22: 02$ \\
Smoking & Unemployed & 32 & 14.68 \\
& Student & 80 & 36.70 \\
Alcohol & Officer & 58 & 26.61 \\
& Yes & 146 & 66.97 \\
Those who have a history of psychiatric application & No & 72 & 33.03 \\
& Yes & 52 & 23.85 \\
& No & 166 & 76.15 \\
& Yes & 25 & 11.47 \\
& None & 193 & 88.53 \\
\hline
\end{tabular}

Table 2. Comparison of SAAS and HAD Scores in Patient and Control Groups

\begin{tabular}{lccccccc}
\hline & & & & & & \\
& & Mean & Std. Dev. & Std. Error & Min. & Max. & $\mathrm{p}$ \\
\multirow{3}{*}{ SAAS } & Patient & 57.11 & 7.55 & 0.66 & 40 & 66 & \multirow{2}{*}{0.001} \\
& Control & 19.27 & 8.88 & 0.94 & 5 & 42 & \\
\multirow{3}{*}{ HADS-A } & Total & 41.66 & 20.32 & 1.37 & 5 & 66 & \\
& Patient & 6.96 & 2.59 & 0.22 & 4 & 18 & \multirow{2}{*}{0.703} \\
& Control & 7.10 & 2.73 & 0.29 & 4 & 15 & \\
HADS-D & Total & 7.02 & 2.65 & 0.18 & 4 & 18 & \\
& Patient & 6.31 & 1.39 & 0.12 & 3 & 9 & 0.001 \\
& Control & $7-29$ & 2.31 & 0.24 & 4 & 17 & \\
\hline
\end{tabular}

SAAS: Social appearance Anxiety scale, HADS-A: Hospital Anxiety and depression scale Anxiety subscale, HADS-D: Hospital Anxiety and depression scale depression subscale

group HAD depression subscale scores were lower than the control group $(p<0.01)$ (Table 2).

Correlation Analysis: The correlation coefficient between the HAD Anxiety subscale and SAAS is 0 . 484 and this relationship was found to be statistically significant $(\mathrm{p}<0.01)$. That is, individuals in the patient group SAAS as HAD Anxiety values increase, the values of HAD also increase Table 3.

The correlation coefficient between the HAD Depression subscale and SAAS was found to be 0.06 , and this relationship was not found to be statistically significant. (Table 3).
The correlation coefficient between the dermatological quality of life index (DLQI) and SAAS was found to be 0.060 and this relationship was not found to be statistically significant (Table 3 ).

The correlation coefficient between Visual analog scale (VAS) and SAAS was found to be 0.28 and this relationship was found to be statistically significant $(p<0.01)$. That is, individuals in the patient group SAAS As the values increase, the VAS values also increase Table 3.

The control group correlation relationship is indicated in Table 3. 
Table 3. Correlations in the patient group

\begin{tabular}{lcccccc}
\hline & Age & SSI score & HADS-A & HADS-D & DYKI & $\begin{array}{c}\text { Visual } \\
\text { Analogue } \\
\text { Scoring }\end{array}$ \\
\hline Age & 1 & & & & \\
SAAS & $0.486 * *$ & 1 & & & \\
HADS-A & 0.155 & $0.484 * *$ & 1 & & \\
HADS-D & $0.365 * *$ & 0.061 & 0.106 & 1 & & \\
DLQI & 0.065 & 0.060 & $0.263 * *$ & 0.006 & 1 & 1 \\
Visual Analogue Scoring & $0.178 *$ & $0.282 * *$ & $0.223 *$ & 0.167 & $0.650 * *$ & 1 \\
\hline *: $<0.05 ; * *:$ p $<0.01$, SAAS: Social appearance Anxiety scale, HADS-A: Hospital Anxiety and depression scale Anxiety \\
subscale, HADS-D: Hospital Anxiety and depression scale depression subscale, DLQI: Dermatological quality of life index
\end{tabular}

\section{Discussion}

In this study, the patient group with contact dermatitis and the healthy control group were compared. It was determined that the Anxiety level of the contact dermatitis group due to social appearances was higher than the healthy individuals. We determined that the lesions in the visible region of the patients significantly increased their social Anxiety levels.

Many dermatological diseases can cause mental disorders in addition to physical findings. While there are many studies on psychiatric morbidity in dermatological patients, the Anxiety levels of contact dermatitis patients due to their social appearance have not been investigated.

The appearance of the skin is important for the individual's self-image and social interaction (11). In addition to physical deterioration, contact dermatitis can be difficult to treat which may cause Anxiety in patients, and ultimately lead to an increase in psychosocial burden with lifestyle changes (12). It has been shown that individuals with lesions in the visible areas of their bodies are associated with a similar decrease in self-esteem as well as negative body image perception (13).

Silverberg's study has shown that skin lesions in older children with contact dermatitis are ridiculed by their peers, and accordingly, they are negatively affected in children's self-perception (17). It has been reported that these skin lesions in school-age children with contact dermatitis may be mistaken that this condition may be contagious, may prevent them from playing with other children, and cause low self-esteem with social withdrawal (18). In this study, individuals with contact dermatitis with external lesions were found to have significantly higher Anxiety levels due to their social appearance compared to the control group. Our study is the first in the literature in this respect.
When the literature is reviewed, it has been emphasized in some reviews that dermatological diseases and the origins of neuropsychiatric diseases are closely related to the diseases related to these two systems, and the role of some hormones and neurotransmitters or neuropeptides is pointed out. For this reason, it is emphasized that these two systems originating from the ectoderm can affect or trigger each other, and a deterioration in one of the systems may cause problems in the other system (19). In their study, Aktan et al. stated that $33.4 \%$ of those with skin disease were accompanied by psychiatric disorders (17).

The social appearance Anxiety scale is a scale that focuses on the Anxiety about how one's body is perceived by other people rather than personal attitudes (18). In the study, no difference was found between the HADS-A scale scores between the two groups, and the SAAS score was higher in the patient group. This indicates that social appearance is a particular source of anxiety in people with contact dermatitis. Various mechanisms exist to try explain the relationship between dermatitis and anxiety. It can be tried in laboratory environments where there is an organic link between stress and dermatitis. Psychosocial stress can exacerbate various dermatological diseases. The stress response of the central nervous system triggers a series of pathways with the activation of the sympathetic nervous system and the release of steroid hormones (19). The hypothaloma-pituitary-adrenal (HPA) axis is triggered by stress and adversely affects skin diseases such as contact dermatitis, psoriasis, urticaria, alopecia areata, and acne (20).

Limitations of the study; Since SAAS and HAD scales are self-report scales, different results may occur depending on the sociocultural levels, perceptions and educational status of the participants. Since the study is a cross-sectional study, more comprehensive studies are needed to make general inferences. However, it is thought that this study is important in 
terms of understanding the relationship between social appearance Anxiety and contact dermatitis and shedding light on similar studies to be done in the future.

In individuals with contact dermatitis; It should be considered as a disease that can affect mental health because it causes Anxiety disorder due to social appearances. While treating the patients with contact dermatitis, mental support should be provided at the same time. Although contact dermatitis is not associated with significant morbidity and mortality or physical disability, it can have significant psychological and social consequences. Restoring the psychosocial effect of contact dermatitis, along with its treatment, can be considered a necessity. With a good contact dermatitis treatment, reducing the Anxiety levels of patients due to their social appearance and strengthening their self-perception can increase the quality of life of patients. Otherwise, the decrease in the quality of life will negatively affect the course of such dermatological disorders, and the increased and prolonged treatment periods will cause problems in terms of social-occupational aspects as well as fatigue in the patients.

\section{Acknowledgements}

The author would like to thank Associate Professor İbrahim Halil Yavuz, who works at the Department of Dermatology at Yüzüncü Yil University, Faculty of Medicine, for his contributions.

\section{References}

1. Thyssen JP, Johansen JD, Linneberg A, Menné T. The epidemiology of hand eczema in the general population-prevalence and main findings. Contact Dermatitis 2010; 62: 75-87.

2. Lima AL, Timmermann V, Illing T, Elsner P. Contact dermatitis in the elderly: predisposing factors, diagnosis, and management. Drugs \& aging 2019; 36(5): 411-417.

3. Šitum M, Kolić M, Buljan M. Psychodermatology. Acta Medica Croat 2016; 70: 35-38.

4. Gupta MA, Gupta AK. Psychiatric and Psychological Co-Morbidity in Patients with Dermatologic Disorders: Epidemiology and Management. C. 4, American Journal of Clinical Dermatology 2003; 833-842.

5. Kieć-Swierczyńska M, Dudek B, Krecisz B, Swierczyńska-Machura D, Dudek W, Garnczarek A, Turczyn K. The role of psychological factors and psychiatric disorders in skin diseases. Medycyna pracy 2006; 57: 551-555.

6. Lahfa M. Définition et psychopathologie de la dermite chronique des mains. Ann Dermatol Venereol 2014; 141.

7. Koo J, Lebwohl A. Psychodermatology: the mind and skin connection. American family physician 2001; 64: 1873.

8. Timms RM. Moderate acne as a potential barrier to social relationships: myth or reality?. Psychology, health \& medicine 2013; 18: 310-320.

9. Ongenae K, Beelaert L, van Geel N, Naeyaert JM. Psychosocial effects of vitiligo. C. 20, Journal of the European Academy of Dermatology and Venereology 2006; 1-8.

10. Duru P, Örsal Ö. The effect of acne on quality of life, social appearance anxiety, and use of conventional, complementary, and alternative treatments. Complementary Therapies in Medicine 2021; 56: 102614.

11. Bell BT, Cassarly JA, Dunbar L. Selfieobjectification: Self-objectification and positive feedback ("likes") are associated with frequency of posting sexually objectifying selfimages on social media. Body image, 2018;26, 83-89.

12. Marron SE, Tomas-Aragones L, Navarro-Lopez J, Gieler U, Kupfer J, Dalgard FJ, Marijuan PC. The psychosocial burden of hand eczema: data from a European dermatological multicentre study. Contact Dermatitis 2018; 78: 406-412.

13. Chinappi JF. The Effects of Chronic Skin Disorders and Ballet on Body Image, Stress, Social Support, and Self-Esteem: A QuasiExperimental Study (Doctoral dissertation, Capella University 2017.

14. Silverberg NB. Typical and atypical clinical appearance of atopic dermatitis. Clinics in dermatology, 2017;35(4), 354-359.

15. Magin P, Adams J, Heading G, Pond D, Smith W. Experiences of appearance-related teasing and bullying in skin diseases and their psychological sequelae: results of a qualitative study. Scandinavian journal of caring sciences 2008; 22: 430-436.

16. Cengiz GF, Gürel G. Difficulties in emotion regulation and quality of life in patients with acne. Quality of Life Research 2020; 29: 431438.

17. Aktan Ş, Özmen E, Şanli B. Psychiatric disorders in patients attending a dermatology outpatient clinic. Dermatology 1998; 197: 230234.

18. Koskina A, Van den Eynde F, Meisel S, Campbell IC, Schmidt U. Social appearance anxiety and bulimia nervosa. Eating and 
Weight Disorders-Studies on Anorexia, Bulimia and Obesity 2011; 16: 142-145.

19. Slominski AT, Zmijewski MA, Zbytek B, Tobin DJ, Theoharides TC, Rivier J. Key role of CRF in the skin stress response system. Endocrine reviews 2013; 34: 827-884.
20. Kim JE, Cho BK, Cho DH, Park HJ. Expression of Hypothalamic-pituitary-adrenal axis in common skin diseases:Evidence of its association with stress-related Disease Activity. C. 93, Acta Dermato-Venereologica. 2013; 387-393. 